\title{
Simultaneous temporally and spectrally resolved Raman coherences with single-shot fs/ns rotational CARS
}

\author{
Ali Hosseinnia, Maria Ruchisan, Pengu Ding, Per-Erik Bengtsson, And \\ JOAKIM BOOD* \\ Division of Combustion Physics, Lund University, Box 118, 22100 Lund, Sweden \\ *Corresponding author: joakim.bood@forbrf.lth.se
}

Manuscript accepted for publication in Optics Letters (Optical Society of America), 2020, https://doi.org/10.1364/OL.380247

\begin{abstract}
A novel technique for studies of the dynamics of molecular coherences has been developed. The concept is based on prompt excitation using broadband femtosecond laser pulses, whereupon a narrowband nanosecond laser pulse probes the fast dynamics of the coherences. Detection of the spectrally dispersed coherent signal using a streak camera allows simultaneous spectrally and temporally resolved studies of all excited coherences in a single-shot acquisition. Here we demonstrate the high capacity and versatility of this hybrid femtosecond/nanosecond coherent anti-Stokes Raman scattering (CARS) technique through some illustrative examples, all single-shot measurements, namely time-resolved studies of rotational Raman coherences in nitrogen and air, dynamics of Stark effect on rotational lines, and beating phenomena originating from close-lying rotational lines.
\end{abstract}

Detailed knowledge about molecular vibrations and rotations is crucial for the understanding of chemical bonds and chemical reaction dynamics. The time scales of molecular vibrations and rotations are ultrashort, on the order of 100 fs and 100 ps, respectively, thus requiring measurement techniques providing extremely high temporal resolution. The advent of ultrashort lasers, i.e. picosecond (ps) and femtosecond (fs) lasers, together with advances in time-resolved spectroscopy, have provided scientists with spectroscopic tools with the capacity to study these fundamental molecular motions. Ultrafast spectroscopy has, for example, enabled studies of transition-state dynamics [1], coherent control of chemical reactions [2], accurate determination of molecular constants [3], broadening of spectral lines [4], and molecular alignment and orientation [5]. Exhaustive reviews of ultrafast spectroscopy and femtochemistry can be found in e.g. [6, 7].

Besides exceptional temporal resolution, ultrashort lasers also provide extremely high peak power and broad spectral coverage, factors that have been pivotal in advancing the performance and versatility of non-linear spectroscopic techniques in particular. Coherent anti-Stokes Raman scattering (CARS) is a very powerful nonlinear technique for molecular detection that has benefited immensely from these laser sources [8]. It is a four-wave mixing technique initiated by two electrical fields, provided by two laser pulses, historically referred to as the pump and Stokes pulses, creating a coherent excitation of molecules. A third electrical field, provided by another laser pulse (the probe pulse), is then interacting with the coherent state and creates a fourth electrical field, called the anti-Stokes pulse or CARS signal, which carries information about Raman resonances from the molecules present in the probe volume.

The CARS technique has been established in a great variety of different configurations, probing either rovibrational or pure rotational Raman resonances, and it has been used for research within a large number of scientific fields as well as industrial applications. Coherent anti-Stokes Raman scattering techniques based on nanosecond (ns) pulses have been continuously developed and applied since the 1970s [8]. Nanosecond CARS offers excellent spectral resolution for molecular detection, and thermometry based on nitrogen $\left(\mathrm{N}_{2}\right)$ can be done with relative accuracy and precision within a few percent [8]. The method, however, suffers from interferences from non-resonant background signals and molecular collisions, whose impact on the spectral shape is difficult to predict since it is dependent on pressure, temperature, and chemical composition. These limitations have motivated development of CARS concepts based on ps and fs pulses, which allow exploitation of the signal in the time domain.

Since lifetimes of the coherently excited states often are on the order of hundreds of picoseconds, the short duration of ps laser pulses permits time separation of the pump/Stokes and probe pulses. Furthermore, the use of broadband ps pulses, created with a modeless dye laser, together with a near-transform-limited ps pulse, allows broadband psCARS with high spectral resolution [9]. Since non-resonant contribution to the CARS signal is present only when all pulses temporally overlap, it is hence possible to eliminate this interference using ps-CARS with a delayed probe pulse [10-12]. Moreover, by varying the delay between the pump/Stokes pulses and the probe pulse, it is possible to measure the decay rate of the probed coherence. Such measurements allow determination of Raman linewidths. Being of great importance for the development of accurate spectroscopic models, Raman linewidths have been the topic of a large number of studies; see e.g. [13-16].

Obviously, even higher temporal resolution can be obtained with $\mathrm{fs}$ laser pulses, but the wide spectral bandwidths of fs pulses rule out the 
possibility to extract molecular information from the spectrally dispersed signal; instead the molecular response has to be analyzed in the time domain. Femtosecond CARS with time-resolved detection allows not only elimination of nonresonant background, but also measurements that are not affected by molecular collisions [17].

In order to take advantage of the high temporal resolution provided by fs pulses, while also obtaining high spectral resolution, various hybrid CARS techniques have been proposed. Pestov et al. [18] developed a concept where fs pulses are used to drive the pump and Stokes transitions, i.e. broadband preparation of the Raman coherences, for which an optimally shaped time-delayed narrowband ps pulse probes the coherences. This approach enables recording of spectrally resolved CARS signal for different probe delays. Initial work on hybrid fs/ps CARS techniques, focusing on biochemical sensing [19], have been followed by extensive development of similar concepts optimized for high-speed gas thermometry $[20,21]$.

Clearly, there are a number of time-resolved CARS concepts available. However, time-resolved studies of Raman coherences are generally carried out by a sequence of measurements with different delays between the pump/Stokes pulses and the probe pulse, which is time consuming and prevents studies under rapidly fluctuating conditions, for which single-shot measurements are required. As an example, a hybrid fs/ps CARS configuration, based on four spatially and temporally separated probe beams, has been demonstrated for singleshot measurements of coherence decay times [22]. Besides being experimentally complex, the low number of probe measurements makes the method less accurate in terms of sensing the shape of the decay curves. Our research group has recently demonstrated an approach for continues time-resolved detection of coherences using a streak camera, where the coherences are generated in a hybrid ps/ns rotational CARS (RCARS) arrangement, i.e. ps pulses for the pump and Stokes transitions and a long (5 ns) pulse for probing [23]. Although studies of the full temporal evolution of spectrally resolved Raman coherences can be made on a single-shot basis, the relatively narrow linewidth of the ps pulses allows only a limited number of coherences (one or two for $\mathrm{N}_{2}$ and $\mathrm{O}_{2}$ ) to be probed within a single acquisition.

Thus, there is presently no technique that allows studies of the full temporal and spectral evolution of Raman coherences on a single-shot basis. In the present work we propose and demonstrate a new approach that has this capability. This approach is based on prompt excitation of Raman coherences using broadband fs laser pulses, after which a narrowband single-mode ns laser pulse probes the coherences, i.e. hybrid fs/ns CARS. Detection of the spectrally dispersed signal using a streak camera enables simultaneous spectrally and temporally resolved measurements of all excited coherences in a single-shotacquisition. The linewidth of the pump and Stokes pulse is $150 \mathrm{~cm}^{-1}$ (FWHM). Thus, the effective spectral bandwidth of the coherent excitation is a convolution between these two pulses, which implies an effective spectral coverage of $210 \mathrm{~cm}^{-1}$ (FWHM). In this letter we demonstrate the exceptional capacity and versatility through some illustrative examples, namely time-resolved studies of rotational Raman coherences in nitrogen and air, beating phenomena due to overlapping nitrogen and oxygen rotational lines, and the dynamics of Stark effect on the rotational lines.

Figure 1a shows a schematic temporal diagram of fs/ns RCARS, where the two overlapping Fourier-limited fs pulses, pump $\left(\omega_{1}\right)$ and Stokes $\left(\omega_{2}\right)$, are positioned at the center of the ns probe pulse $\left(\omega_{3}\right)$. Spatially the laser pulses overlap and the CARS signal, $\omega_{\text {CARS, is created }}$ obeying the phase-matching condition of $\omega_{\text {CARS }}=\omega_{1}-\omega_{2}+\omega_{3}$. The experimental setup is schematically outlined in Fig. $1 \mathrm{~b}$.

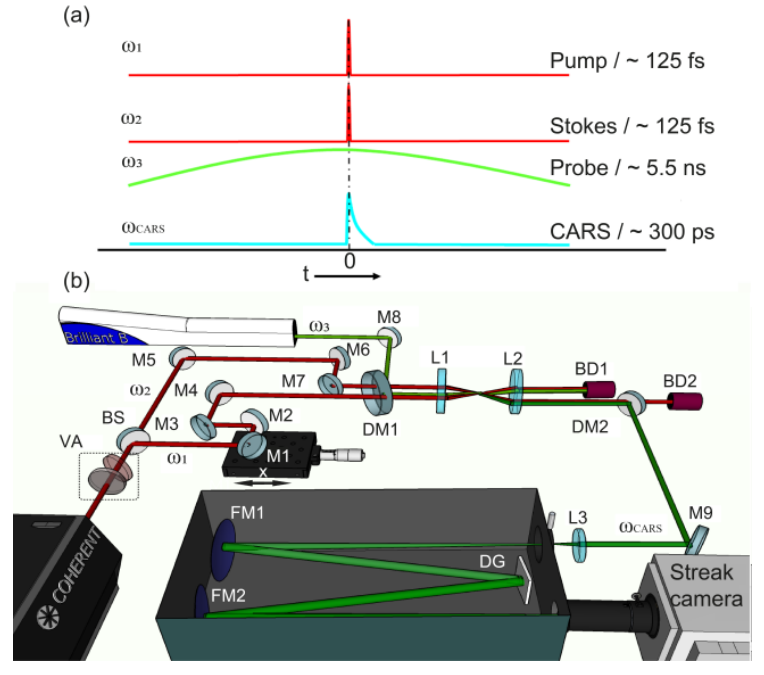

Fig. 1 (a) Temporal diagram of fs/ns RCARS, and (b) schematic of the experimental setup; VA: variable attenuator, M1-9: mirrors, DM1,2: dichroic mirrors, BD1,2: beam dumps, L1-3: spherical lenses, FM1,2: focusing mirrors, and DG: diffraction grating.

A Ti:Sapphire laser system (Coherent, Hidra-50) was used to deliver 125 -fs laser pulses at $800 \mathrm{~nm}$ with $10 \mathrm{~Hz}$ repetition rate. A beam splitter divides the fs pulse into two of equal energy, providing $\omega_{1}$ and $\omega_{2}$. A variable attenuator was employed to lower the fs pulse energies from 2 $\mathrm{mJ} /$ pulse, at the output of the laser, to a specific value depending on the application. A 10-Hz single-mode Nd:YAG laser (Quantel, Brilliant b) delivered laser pulses with 5.5 ns duration full-width-half-maximum (FWHM), a wavelength of $532 \mathrm{~nm}$, and linewidth $0.005 \mathrm{~cm}^{-1}$ (FWHM), providing probe pulses $\left(\omega_{3}\right)$ with an average pulse energy of $40 \mathrm{~mJ}$ in the probe volume.

The $\omega_{1}$ pulse was directed into an optical delay line, after which it was superimposed onto $\omega_{3}$ and travelling parallel to $\omega_{2}$ separated by 2 $\mathrm{cm}$. All three pulses were focused with a spherical lens (L1, $f_{1}=500 \mathrm{~mm}$ ) so that they overlapped temporally and spatially in a planar BOXCARS phase-matching configuration inside a cell with optical access but without windows [24]. The generated CARS signal ( $\omega$ CARS) was collimated by another spherical lens (L2, $f_{2}=300 \mathrm{~mm}$ ), separated from $\omega_{2}$ by a dichroic mirror, and focused onto the entrance slit of a 1-meter Czerny-Turner spectrograph by a spherical lens (L3). The focal length of L3 was $750 \mathrm{~mm}$ in all measurements except in the study of Stark effect, reported towards the end of the letter, where it was $150 \mathrm{~mm}$. The signal was then dispersed by a holographic grating $(2,400$ grooves $/ \mathrm{mm})$ and imaged onto the slit of the streak camera (Optronis, Optoscope). Singleshot RCARS signals were recorded with a streak rate of $50 \mathrm{ps} / \mathrm{mm}$, resulting in 2D images with time and wavelength along the vertical and horizontal axes, respectively. The dispersion was $0.12 \mathrm{~cm}^{-1} /$ pixel, and the spectral resolution was $2.8 \mathrm{~cm}^{-1}$. The temporal resolution, determined by recording a purely nonresonant RCARS signal in argon (Fig. 2b), was 22 ps. Synchronization between the laser pulses $\left(\omega_{1}, \omega_{2}\right.$ and $\omega_{3}$ ) and the streak camera was established using two pulse/delay generators (SRS, DG535, and Coherent, SDG Elite), resulting in a temporal jitter with a root-mean square-of 45 ps between the streak camera and the CARS signal.

Figure 2a illustrates a simultaneous spectrally and temporally resolved pure RCARS signal of $\mathrm{N}_{2}$ recorded in a single-shot acquisition. The signal was obtained with a pulse energy of $\sim 5 \mu \mathrm{J}$ for each of the fs pulses. To compensate for the finite spectral bandwidth of $\omega_{1}$ and $\omega_{2}$, all spectra were divided in the spectral domain by a nonresonant spectrum recorded in argon; shown in Fig. $2 \mathrm{~b}$. 


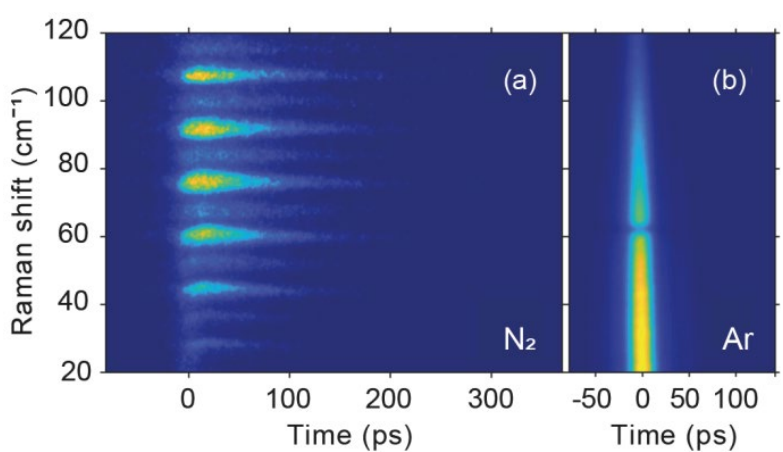

Fig. 2 (a) Normalized spectrally and temporally resolved single-shot RCARS signal of $\mathrm{N}_{2}$ at ambient conditions. (b) Rotational CARS signal of argon recorded under the same condition, and averaged over 500 single shots. The apparent line in the argon signal at $\sim 60 \mathrm{~cm}^{-1}$ is due to imperfections in the detection system.

The coherences deteriorate via collisional dephasing in a few hundred picoseconds at ambient conditions. However, the applied streak rate of the detector allowed capturing of $\sim 1$ ns dynamics of the signal to make sure the entire signal is recorded despite temporal jitter.

The $\mathrm{N}_{2}$ coherences decay with a first-order exponential rate as illustrated in the logarithmic plot for the S(4),S(8), and S(12) lines in Fig. $3 a$, together with the corresponding decay constant $(1 / \mathrm{e}), \tau$, obtained from first-order polynomial fits. Time zero is chosen to be when CARS signals are maximized due to temporal overlap of $\omega_{1}$ and $\omega_{2}$. At $t \approx 27$ ps, where the polynomial fits start, the nonresonant contribution of the signal, indicated by the dash-dotted line, has decreased by two orders of magnitude, while the resonant part of the signal has not experienced any significant signal loss. The time extent during which the measured signal is influenced by nonresonant contribution is set by the temporal resolution of the instrument. Moreover, assuming optimized slit widths for the spectrometer and streak camera, the spectral and temporal resolutions are inversely related, since higher number of illuminated grooves on the grating (higher spectral resolution), results in higher temporal dispersion and hence lower time resolution, and vice versa [25]. However, the setup allows easy adjustment of the temporal and spectral resolutions, by the choice of the focal length of L3, based on the specific application.

The Raman linewidth, $\Gamma$, is inversely proportional to the coherence decay constant, $\tau$, i.e. $\Gamma_{\mathrm{J}}=(2 \pi \mathrm{c} \tau)^{-1}[13]$, where $c$ is the speed of light and the subscript $J$ designates the lower rotational state of the transition. Using this expression, the Raman linewidths of the S-branch of $\mathrm{N}_{2}$ were calculated from the measured decay rates for $J=2-13$, both from singleshot and averaged spectra. The results are depicted in Fig. $3 \mathrm{~b}$. The blue empty circles are median values of the linewidths extracted from 500 recorded single-shots with $1-\sigma$ error bars. The standard deviation averaged over all lines is $0.012 \mathrm{~cm}^{-1}$. The red squares are linewidths evaluated from the averaged spectrum of the same 500 single shots. Both results show good agreement with previously reported experimental data (orange stars) [14]. Additionally, the comparison with two well-established theoretical models; modified exponential gap (MEG) [26] and energy corrected sudden (ECS) [27], substantiates the validity of the method.

The ability of the technique to measure Raman linewidths experimentally on a single-shot basis is a prodigious advantage in gasphase thermometry, which eradicates the limitations and uncertainties set by the necessary pre-knowledge about the collider molecules and their number densities in the probe volume. This advantage becomes more prominent at higher pressures, where the effects of collisional dephasing cannot be ignored even at very short delays [4].
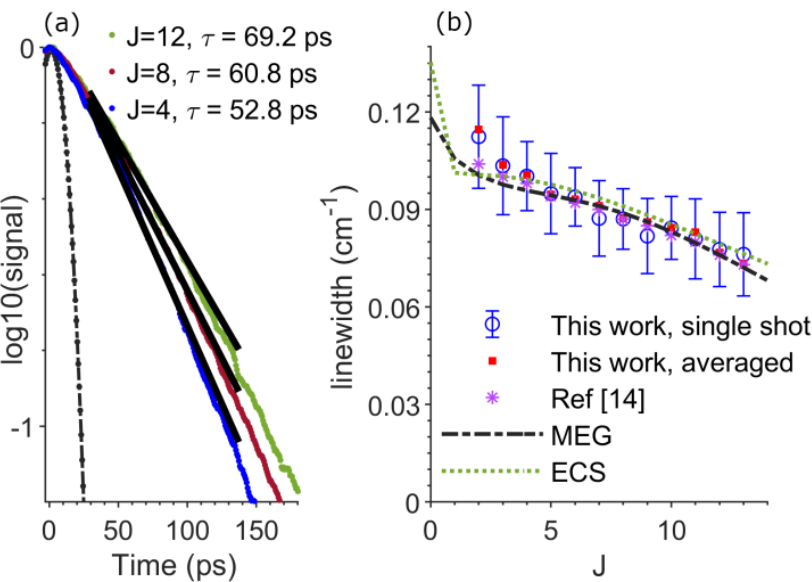

Fig. 3 (a) Time resolved CARS signal of three $\mathrm{N}_{2}$ rotational lines, and (b) extracted S-branch self-broadened $\mathrm{N}_{2}$ Raman linewidths from single shots and averaged spectra of this work together with a previous experimental study [14] and two theoretical models; MEG [26] and ECS [27].

The impact of the method on accuracy and precision in gas-phase thermometry is currently investigated and will be reported in a forthcoming publication.

A spectrogram of RCARS signal recorded in room air is displayed in Fig. 4a. Since air contains $\sim 79 \% \mathrm{~N}_{2}$ and $\sim 21 \% \mathrm{O}_{2}$, the spectrogram contains temporally resolved spectral lines from both molecules. For pairs of $\mathrm{N}_{2}$ and $\mathrm{O}_{2}$ lines that are not spectrally resolved, sinusoidal oscillations, i.e. beats, are superimposed on the exponential decays, as can be seen for the partially overlapping $\mathrm{N}_{2}-\mathrm{S}(12)$ and $\mathrm{O}_{2}-\mathrm{S}(17)$ lines shown in Fig. 4b. The origin of the beat is optical heterodyning, i.e. two optical signals of slightly different frequencies are mixed while entering the detector pixels, which creates an output signal with an amplitude modulation at the difference frequency. A fit of a function consisting of three terms, two single-exponential functions, corresponding to the individual decays of the two lines, and a cross-term containing the sinusoidal beat, is indicated by the red line in Fig. $4 \mathrm{~b}$. The fitted curve corresponds to a beat period of $27.73 \pm 0.05 \mathrm{ps}$, corresponding to a line separation of $1.201 \pm 0.002 \mathrm{~cm}^{-1}$, which is in excellent consistency with the value calculated $\left(1.196 \mathrm{~cm}^{-1}\right)$ based on the most accurate molecular constants for $\mathrm{N}_{2}$ and $\mathrm{O}_{2}$ [28]. This result suggests that the method could be useful for precise measurement of spectral line positions, and, hence, more accurate molecular constants.
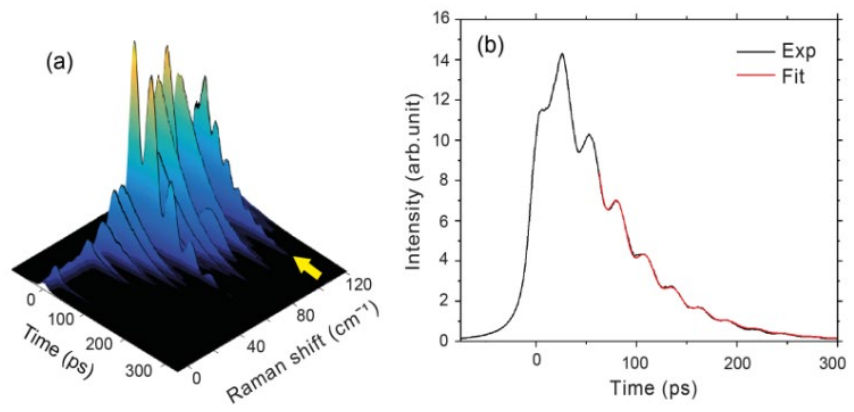

Fig. 4 (a) Spectrogram of averaged rotational CARS signal recorded in ambient air. Frequency beating is evident on several partially overlapping $\mathrm{N}_{2} / \mathrm{O}_{2}$ lines. The partially overlapping $\mathrm{S}(12)$ of $\mathrm{N}_{2}$ and $\mathrm{S}(17)$ of $\mathrm{O}_{2}$, indicated by the arrow, is shown in (b) together with a fit. 


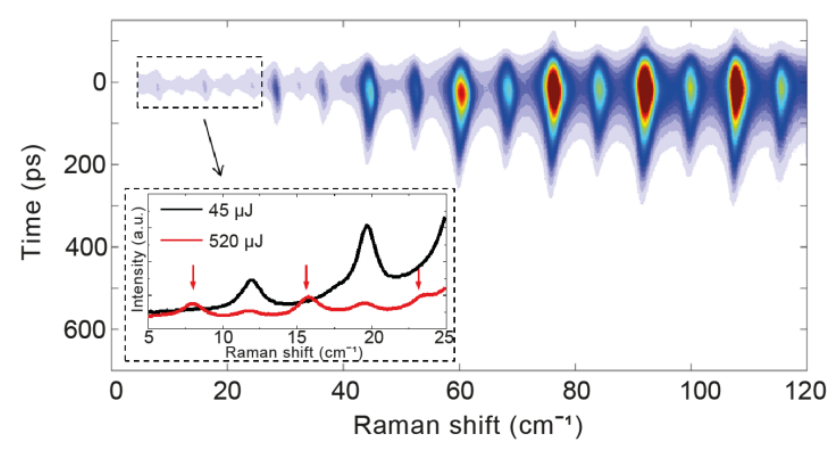

Fig. 5 Spectrogram recorded in $\mathrm{N}_{2}$ with $520 \mu \mathrm{J}$ fs-pulse energy. The lowJ rotational lines are influenced by Stark effect, which is more evident in the time-integrated spectrum (red line), where the peaks indicated by arrows are due to Stark splitting. A spectrum recorded with $45 \mu \mathrm{J}$ pulse energy is shown for comparison (black line).

A set of measurements were carried out with varying pulse energies of the fs laser to study the RCARS signal appearance, spectrally and temporally, by the presence of high electrical fields. The spectral resolution was optimized by using a lens (L3 in Fig. 1b) that matches the f-number of the spectrometer. As mentioned previously, this implies lower temporal resolution (85 ps). Figure 5 shows a spectrogram recorded in $\mathrm{N}_{2}$ with $520 \mu \mathrm{J}$ fs laser pulse energy (pump and Stokes energies added) at the probe volume. The most obvious change, while increasing the fs-laser pulse energy, is that additional spectral lines appear at low Raman shifts, as can be seen in the time-integrated spectrum (red line). The time integration was performed over the final tail of the signal in order to minimize influence from non-resonant contribution. The additional lines, indicated by red arrows, are due to Stark splitting [29]. For comparison; a spectrum recorded at $45 \mu \mathrm{J}$, black line in Fig. 5, does not exhibit additional spectral lines. It was found that Stark effect is not observable for pulse energies below $100 \mu \mathrm{J}$, which corresponds to a mean laser intensity of $1.0 \mathrm{TW} / \mathrm{cm}^{2}$. For most experiments we used much lower fs pulse energies, $15 \mu \mathrm{J}$, in order to stay below the saturation level of the detection system. Hence, due to the very strong CARS signals we foresee that the presented technique also can be used for single-shot measurements at typical flame temperatures. It should be noted that the dynamic range of the streak camera is only 1000:1. However, since the decay evaluation can start already after $27 \mathrm{ps}$ for our case with fs excitation, a signal decay over one order of magnitude is sufficient to establish the decay time constant. Additionally, flatfield and linearity compensation for the streak camera are crucial for achieving reliable quantitative data [30].

It is evident that the newly developed technique opens the door to a multitude of fundamental molecular studies, previously not possible to perform or requiring stable repeatable conditions allowing conventional pump-probe concepts to be used. Equally important, our results also suggest that diagnostics with RCARS in, for example reactive flow applications, can be significantly improved, overcoming the major hurdle due to limited knowledge of Raman linewidths. The method may be particularly useful for studies of plasma discharge dynamics, where the fast stochastic and non-repetitive events require high temporal resolution and single-shot studies.

Funding. European Research Council (ERC), Knutand Alice Wallenberg Foundation, Swedish Foundation for Strategic Research (SSF), and the Swedish Energy Agency through the project GRECOP.

Acknowledgment. We thank Dr. Hans Seyfried, Tetra Pak, Lund, for lending a laser, and Prof. Marcus Aldén for fruitful discussions.
Disclosures. The authors declare no conflicts of interest

\section{REFERENCES}

1. M. Motzkus, S. Pedersen, and A. Zewail, J. Phys. Chem. 100, 5620 (1996).

2. E. Potter, J. Herek, S. Pedersen, Q. Liu, and A. Zewail, Nature 355, 66 (1992).

3. R. T. Carter, and J. R. Huber, Chem. Soc. Rev. 29, 305 (2000).

4. J. D. Miller, S. Roy, J. R. Gord, and T. R. Meyer, J. Chem. Phys. 135, 201104 (2011).

5. O. Ghafur, A. Rouzée, A. Gijsbertsen, W. K. Siu, S. Stolte, and M. J. Vrakking, Nat. Physics 5, 289 (2009).

6. M. Dantus, "Coherent nonlinear spectroscopy: from femtosecond dynamics to control," Ann. Rev. Phys. Chem. 52, 639 (2001).

7. A. K. Patnaik, I. Adamovich, J. R. Gord, and S. Roy, Plasma Sources Sci. Technol. 26, 103001 (2017).

8. S. Roy, J. R. Gord, and A. K. Patnaik, Progr. Energy Combust. Sci. 36, 280 (2010)

9. S. Roy, T. R. Meyer, and J. R. Gord, Opt. Lett. 30, 3222 (2005).

10. S. Roy, T. R. Meyer, and J. R. Gord, Appl. Phys. Lett. 87, 264103 (2005).

11. T. R. Meyer, S. Roy, and J. R. Gord, Appl. Spectrosc. 61, 1135 (2007).

12. A. Bohlin, E. Nordström, B. Patterson, P.-E. Bengtsson, and C. Kliewer, J. Chem. Phys. 137, 074302 (2012).

13. W. D. Kulatilaka, P. S. Hsu, H. U. Stauffer, J. R. Gord, and S. Roy, Appl. Phys. Lett. 97, 081112 (2010).

14. C. J. Kliewer, A. Bohlin, E. Nordström, B. D. Patterson, P.-E. Bengtsson, and T. B. Settersten, Appl. Phys. B 108, 419 (2012). 15. S. Roy, P. S. Hsu, N. Jiang, J. R. Gord, W. D. Kulatilaka, H. U. Stauffer, and J. R. Gord, J. Chem. Phys. 138, 024201 (2013).

16. C. Meißner, J. I. Hölzer, and T. Seeger, Appl. Opt. 58, C47 (2019). 17. R. P. Lucht, S. Roy, T. R. Meyer, and J. R. Gord, Appl. Phys. Lett. 89, 251112 (2006).

18. D. Pestov, R. K. Murawski, G. O. Ariunbold, X. Wang, M. Zhi, A. V. Sokolov, V. A. Sautenkov, Y. V. Rostovtsev, A. Dogariu, and Y. Huang, Science 316, 265 (2007).

19. D. Pestov, X. Wang, G. O. Ariunbold, R. K. Murawski, V. A.

Sautenkov, A. Dogariu, A. V. Sokolov, and M. O. Scully, Proc. Nat. Acad. Scie. 105, 422 (2008).

20. J. D. Miller, M. N. Slipchenko, T. R. Meyer, H. U. Stauffer, and J. R. Gord, Opt. Lett. 35, 2430 (2010).

21. J. D. Miller, S. Roy, M. N. Slipchenko, J. R. Gord, and T. R. Meyer, Opt. Expr. 19, 15627 (2011).

22. B. D. Patterson, Y. Gao, T. Seeger, and C. J. Kliewer, Opt. Lett. 38, 4566 (2013).

23. E. Nordström, A. Hosseinnia, C. Brackmann, J. Bood, and P.-E. Bengtsson, Opt. Lett. 40, 5718 (2015).

24. A. C. Eckbreth, Laser diagnostics for combustion temperature and species (CRC Press, 1996).

25. A. Visco, R. Drake, D. Froula, S. Glenzer, and B. Pollock, Rev. Sci. Instr. 79, 10F545 (2008).

26. M. Koszykowski, L. Rahn, R. Palmer, and M. Coltrin, J. Phys. Chem. 91, 41 (1987).

27. A. E. DePristo, S. D. Augustin, R. Ramaswamy, and H. Rabitz, J. Chem. Phys. 71, 850 (1979).

28. R. R. Laher, and F. R. Gilmore, J. Phys. Chem. Ref. Data 20, 685 (1991).

29. R. Farrow, and L. Rahn, Phys. Rev. Lett. 48, 395 (1982).

30. D. S. Montgomery, R. P. Drake, B. A. Jones, and J. D. Wiedwald, Flat-Field Response And Geometric Distortion Measurements Of Optical Streak Cameras, Proc. SPIE 0832 (SPIE, 1988). 


\section{FULL REFERENCES}

1. M. Motzkus, S. Pedersen, and A. Zewail, "Femtosecond Real-Time Probing of Reactions. 19. Nonlinear (DFWM) Techniques for Probing Transition States of Uni- and Bimolecular Reactions", The Journal of Physical Chemistry 100, 5620-5633 (1996).

2. E. Potter, J. Herek, S. Pedersen, Q. Liu, and A. Zewail, "Femtosecond laser control of a chemical reaction", Nature 355, 66-68 (1992).

3. R. T. Carter, and J. R. Huber, "Quantum beat spectroscopy in chemistry", Chemical Society Reviews 29, 305-314 (2000).

4. J. D. Miller, S. Roy, J. R. Gord, and T. R. Meyer, "Communication: Timedomain measurement of high-pressure $\mathrm{N}_{2}$ and $\mathrm{O}_{2}$ self-broadened linewidths using hybrid femtosecond/picosecond coherent anti-Stokes Raman scattering", The Journal of Chemical Physics 135, 201104 (2011).

5. O. Ghafur, A. Rouzée, A. Gijsbertsen, W. K. Siu, S. Stolte, and M. J. Vrakking, "Impulsive orientation and alignment of quantum-state-selected NO molecules", Nature Physics 5, 289 (2009).

6. M. Dantus, "Coherent Nonlinear Spectroscopy: From Femtosecond Dynamics to Control", Annual Review of Physical Chemistry 52, 639-679 (2001).

7. A. K. Patnaik, I. Adamovich, J. R. Gord, and S. Roy, "Recent advances in ultrafast-laser-based spectroscopy and imaging for reacting plasmas and flames", Plasma Sources Science and Technology 26, 103001 (2017).

8. S. Roy, J. R. Gord, and A. K. Patnaik, "Recent advances in coherent antiStokes Raman scattering spectroscopy: Fundamental developments and applications in reacting flows", Progress in Energy and Combustion Science 36, 280-306 (2010).

9. S. Roy, T. R. Meyer, and J. R. Gord, "Broadband coherent anti-Stokes Raman scattering spectroscopy of nitrogen using a picosecond modeless dye laser", Optics Letters 30, 3222-3224 (2005).

10. S. Roy, T. R. Meyer, and J. R. Gord, "Time-resolved dynamics of resonant and nonresonant broadband picosecond coherent anti-Stokes Raman scattering signals", Applied Physics Letters 87, 264103 (2005).

11. T. R. Meyer, S. Roy, and J. R. Gord, "Improving Signal-To-Interference Ratio in Rich Hydrocarbon-Air Flames Using Picosecond Coherent Anti-Stokes Raman Scattering", Applied Spectroscopy 61, 1135-1140 (2007).

12. A. Bohlin, E. Nordström, B. Patterson, P.-E. Bengtsson, and C. Kliewer, "Direct measurement of S-branch $\mathrm{N}_{2}-\mathrm{H}_{2}$ Raman linewidths using time-resolved pure rotational coherent anti-Stokes Raman spectroscopy", The Journal of Chemical Physics 137, 074302 (2012).

13. W. D. Kulatilaka, P. S. Hsu, H. U. Stauffer, J. R. Gord, and S. Roy, "Direct measurement of rotationally resolved H2 Q-branch Raman coherence lifetimes using time-resolved picosecond coherent anti-Stokes Raman scattering", Applied Physics Letters 97, 081112 (2010).

14. C. J. Kliewer, A. Bohlin, E. Nordström, B. D. Patterson, P.-E. Bengtsson, and T. B. Settersten, "Time-domain measurements of S-branch $\mathrm{N}_{2}-\mathrm{N}_{2}$ Raman linewidths using picosecond pure rotational coherent anti-Stokes Raman spectroscopy", Applied Physics B 108, 419-426 (2012).

15. S. Roy, P. S. Hsu, N. Jiang, J. R. Gord, W. D. Kulatilaka, H. U. Stauffer, and J. R. Gord, "Direct measurements of collisionally broadened Raman linewidths of $\mathrm{CO}_{2}$ S-branch transitions", The Journal of Chemical Physics 138, 024201 (2013).

16. C. Meißner, J. I. Hölzer, and T. Seeger, "Determination of $\mathrm{N}_{2}-\mathrm{N}_{2}$ and $\mathrm{N}_{2}-\mathrm{O}_{2}$ S-branch Raman linewidths using time-resolved picosecond pure rotational coherent anti-Stokes Raman scattering", Applied Optics 58, C47-C54 (2019).

17. R. P. Lucht, S. Roy, T. R. Meyer, and J. R. Gord, "Femtosecond coherent antiStokes Raman scattering measurement of gas temperatures from frequencyspread dephasing of the Raman coherence", Applied Physics Letters 89, 251112 (2006).

18. D. Pestov, R. K. Murawski, G. O. Ariunbold, X. Wang, M. Zhi, A. V. Sokolov, V. A. Sautenkov, Y. V. Rostovtsev, A. Dogariu, and Y. Huang, "Optimizing the
Laser-Pulse Configuration for Coherent Raman Spectroscopy", Science 316, 265-268 (2007).

19. D. Pestov, X. Wang, G. O. Ariunbold, R. K. Murawski, V. A. Sautenkov, A. Dogariu, A. V. Sokolov, and M. O. Scully, "Single-shot detection of bacterial endospores via coherent Raman spectroscopy", Proceedings of the National Academy of Sciences 105, 422-427 (2008).

20. J. D. Miller, M. N. Slipchenko, T. R. Meyer, H. U. Stauffer, and J. R. Gord, "Hybrid femtosecond/picosecond coherent anti-Stokes Raman scattering for high-speed gas-phase thermometry", Optics Letters 35, 2430-2432 (2010).

21. J. D. Miller, S. Roy, M. N. Slipchenko, J. R. Gord, and T. R. Meyer, "Singleshot gas-phase thermometry using pure-rotational hybrid femtosecond/picosecond coherent anti-Stokes Raman scattering", Optics Express 19, 15627-15640 (2011).

22. B. D. Patterson, Y. Gao, T. Seeger, and C. J. Kliewer, "Split-probe hybrid femtosecond/picosecond rotational CARS for time-domain measurement of S-branch Raman linewidths within a single laser shot", Optics Letters 38, 45664569 (2013).

23. E. Nordström, A. Hosseinnia, C. Brackmann, J. Bood, and P.-E. Bengtsson, "Raman linewidth measurements using timeresolved hybrid picosecond/nanosecond rotational CARS", Optics Letters 40, 5718-5721 (2015).

24. A. C. Eckbreth, Laser diagnostics for combustion temperature and species (CRC Press, 1996).

25. A. Visco, R. Drake, D. Froula, S. Glenzer, and B. Pollock, "Temporal dispersion of a spectrometer", Review of Scientific Instruments 79, 10 F545 (2008).

26. M. Koszykowski, L. Rahn, R. Palmer, and M. Coltrin, "Theoretical and experimental studies of high-resolution inverse Raman spectra of molecular nitrogen at 1-10 atm", Journal of Physical Chemistry 91, 41-46 (1987).

27. A. E. DePristo, S. D. Augustin, R. Ramaswamy, and H. Rabitz, "Quantum number and energy scaling for nonreactive collisions", The Journal of Chemical Physics 71, 850-865 (1979).

28. R. R. Laher and F. R. Gilmore, "Improved Fits for the Vibrational and Rotational Constants of Many States of Nitrogen and Oxygen", Journal of Physical and Chemical Reference Data 20, 685-712 (1991).

29. R. Farrow and L. Rahn, "Optical Stark Splitting of Rotational Raman Transitions", Physical Review Letters 48, 395-398 (1982).

30. D. S. Montgomery, R. P. Drake, B. A. Jones, and J. D. Wiedwald, "Flat-Field Response And Geometric Distortion Measurements Of Optical Streak Cameras", Proc. SPIE 0832 (SPIE, 1988). 